\title{
A Modified Internal Model Control for an Unstable Plant with an Integrator in Continuous-Time System
}

\author{
H. Shibasaki, J. Endo, Y. Hikichi, R. Tanaka, K. Kawaguchi, and Y. Ishida
}

\begin{abstract}
In this paper, a design of a modified Internal Model Control for an unstable plant with an integrator in continuous-time system is proposed. The system becomes a stable system by stabilizing an unstable plant and it does not cause a steady-state error by an input side disturbance for an unstable plant with an integrator. We describe details of the proposed method including a design of stabilizing an unstable plant, a design of a disturbance compensator, and a design of whole system. In the simulation study, these simulation results are shown that the proposed method has a superior performance. The nominal plant and the plant with a modeling error can be stabilized and the proposed method can reduce influence by in an input side disturbance of an unstable plant.
\end{abstract}

Index Terms-Internal model control, unstable plant, disturbance compensator.

\section{INTRODUCTION}

Internal Model Control (IMC) [1]-[5] and Smith Predictor [6]-[7] are known as effective methods to control a plant. However, a system causes a steady-state error by an input side disturbance for a plant with an integrator. Astrom et al. [8] proposed new Smith predictor with a superior performance. However, this method has a problem that parameter adjustments are complicated. Authors [9] proposed the discrete modified Internal Model Control. This method does not cause a steady-state error by an input side disturbance for a plant with an integrator.

Besides, Internal Model Control and Smith Predictor cannot control an unstable plant. Therefore, the whole system is not stabilized for an unstable plant. For this problem, Yamada [10] proposed Modified Internal Model Control. This method stabilizes an unstable plant and becomes a stable system. However, this method causes a steady-state error by an input side disturbance for an unstable plant with an integrator.

From the above problems, in this paper, we propose the modified Internal Model Control for an unstable plant with an integrator in continuous-time system. This proposed method stabilizes an unstable system, and introduced disturbance compensator does not cause a steady-state error by an input side disturbance for an unstable plant with an integrator.

Manuscript received August 2, 2012; revised September 6, 2012

The authors are with the School of Science and Technology, Meiji University, Kawasaki-shi, Kanagawa, 214-8571 JAPAN (e-mail: ce11045@ meiji.ac.jp).
II. INTERNAL MODEL CONTROL (IMC)

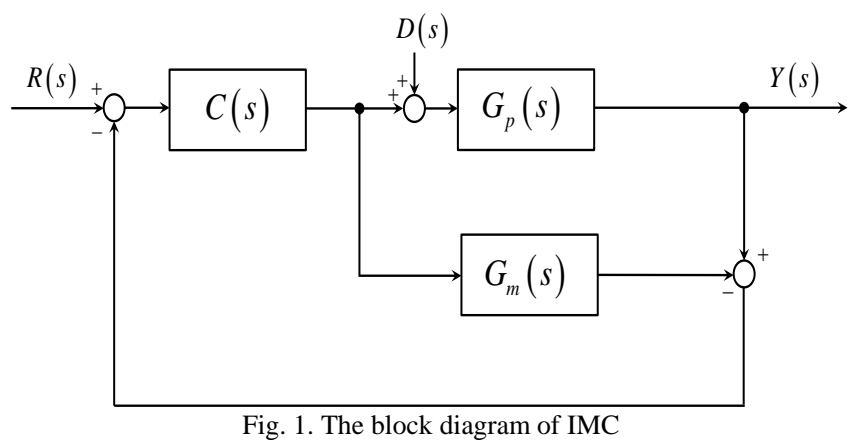

Internal Model Control (IMC) is the method=based on a plant model to build the controller. Fig. 1 is the block diagram of IMC.

$R(s), Y(s)$ and $D(s)$ are an input value, an output value, and an input-side disturbance, respectively. And $G_{p}(s)$ and $G_{m}(s)$ are a plant and a plant model, where these are of equal relationship. $C(s)$ is a controller. From Fig.1, the target-value response and the disturbance response are written by

$$
\begin{gathered}
\frac{Y(s)}{R(s)}=\frac{C(s) G_{p}(s)}{1+C(s) G_{p}(s)-C(s) G_{m}(s)}=C(s) G_{p}(s) \\
\frac{Y(s)}{D(s)}=\frac{G_{p}(s)\left\{1-C(s) G_{m}(s)\right\}}{1+C(s) G_{p}(s)-C(s) G_{m}(s)} \\
=G_{p}(s)\left\{1-C(s) G_{m}(s)\right\}
\end{gathered}
$$

IMC is the effective method to control a plant. However, when a plant is an unstable plant, a system is not a stable system. And if an integral plant has an input side disturbance, it causes a steady-state error. Therefore, we propose a method to solve these problems. From the next section, it describes the detail of the proposed method.

\section{PROPOSED METHOD}

In this section, we describe the proposed method which stabilizes an unstable system and does not cause a steady-state error by an input side disturbance for a plant with an integrator. Fig. 2 is the block diagram of proposed method.

$R(s), Y(s)$ and $D(s)$ are a reference value, an output value, and an input side disturbance, respectively.

$G_{p}(s)$ is an unstable plant with an integrator, $G_{m}(s)$ is a plant model. Here, $G_{p}(s)$ and $G_{m}(s)$ are of equal relationship. $G_{p}(s)$ is resolved by a stable term and unstable term. It means that $G_{p}(s)=G_{m}(s)=G_{s t}(s) G_{u n}(s) . K(s)$ is a stabilization controller, and $C(s)$ is a controller, $C(s)=C_{m}(s)\left\{G_{m}(s)\right.$ 
$\left./ G_{u n}(s)\right\}^{-1}$. Where, $C_{m}(s)$ is a reference model. $M(s)$ is a disturbance compensator. $G(s)$ is a stable system by $K(s)$ which is a stabilization controller to stabilize an unstable plant. In this paper, $G_{p}(s)$ is an unstable plant with an integrator, thus, $K(s)$ becomes a differential controller.

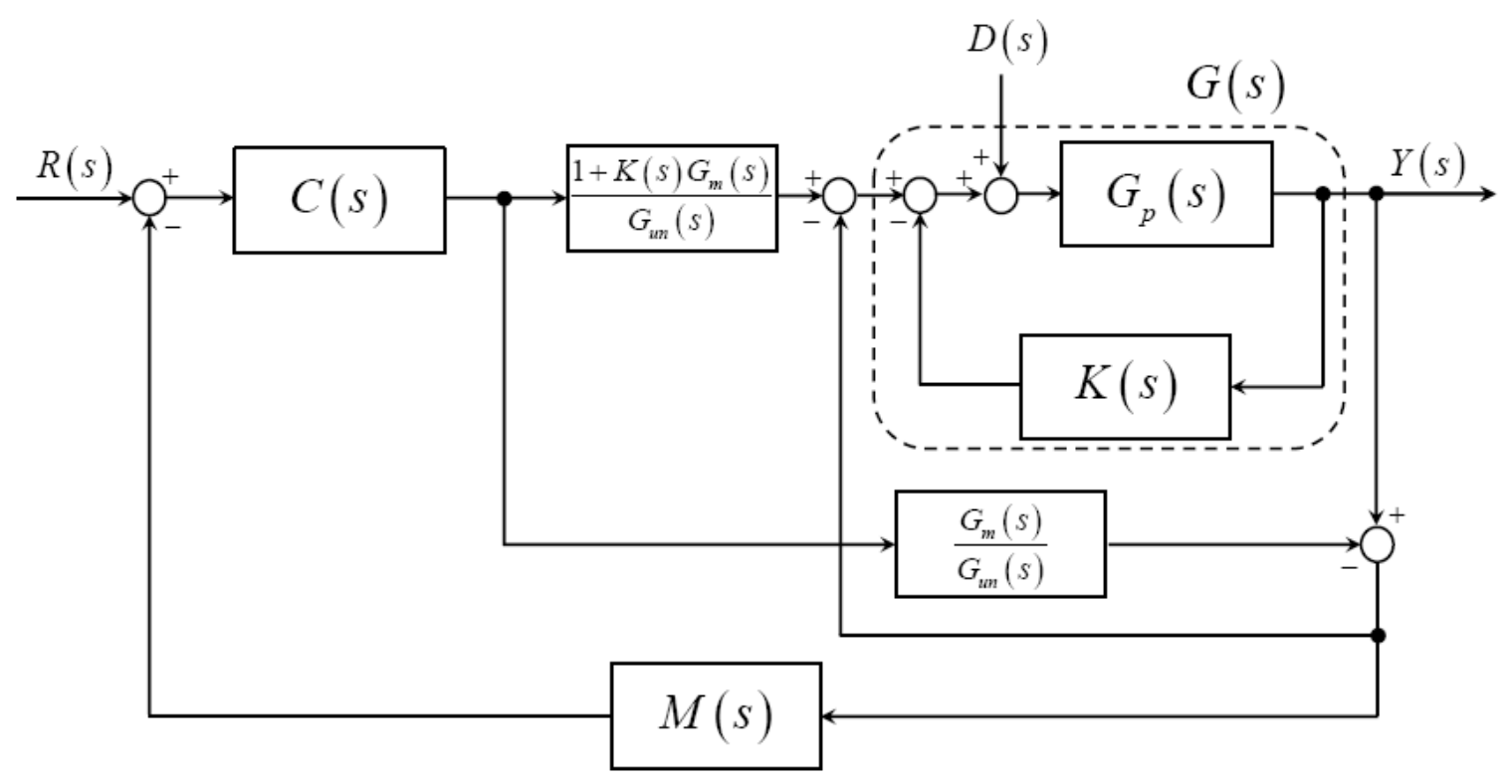

Fig. 2. The block diagram of proposed method

The design method of $G(s)$ is as follows.

$$
\begin{gathered}
G_{p}(s)=\frac{b}{s(s-a)} \\
K(s)=k s \\
G(s)=\frac{G_{p}(s)}{1+G_{p}(s) K(s)}=\frac{b}{s\{s+(b k-a)\}}
\end{gathered}
$$

$G(s)$ becomes the stable system if $b k>a$.

Then, we design $1+G_{m}(s) K(s) / G_{u n}(s)$ which is designed by Yamada's method [10] as shown in Eq. 6. However, this equation is not proper.

$$
\begin{aligned}
\frac{1+G_{m}(s) K(s)}{G_{u n}(s)} & =\frac{s^{2}+(b k-a) s}{b s} \\
& =\frac{s+(b k-a)}{b}
\end{aligned}
$$

Therefore, we redesign Eq. 6 as Eq. 7.

$$
\begin{aligned}
\frac{1+G_{m}(s) K(s)}{G_{u n}(s)} & =\frac{s+(b k-a)}{b} \frac{1}{s} \\
& =\frac{s+(b k-a)}{b s}
\end{aligned}
$$

From this reason, $G_{m}(s) / G_{u n}(s)$ is written as follows

$$
\begin{aligned}
\frac{G_{m}(s)}{G_{u n}(s)} & =\frac{1}{s} \frac{1}{s} \\
& =\frac{1}{s^{2}}
\end{aligned}
$$

Furthermore, $C(s)=C_{m}(s)\left\{G_{m}(s) / G_{u n}(s)\right\}^{-1}$, and $C_{m}(s)$ are given by Eq. 9 and Eq.10.

$$
C_{m}(s)=\frac{\omega_{n}^{2}}{s^{2}+2 \zeta \omega_{n} s+\omega_{n}^{2}}
$$

$$
\begin{aligned}
C(s) & =C_{m}(s)\left\{\frac{G_{m}(s)}{G_{u n}(s)}\right\}^{-1} \\
& =C_{m}(s) s^{2} \\
& =\frac{\omega_{n}{ }^{2} s^{2}}{s^{2}+2 \zeta \omega_{n} s+\omega_{n}^{2}}
\end{aligned}
$$

From Fig.2, the target-value response is written as follows

$$
\frac{Y(s)}{R(s)}=C_{m}(s)
$$

This is the model following control system. On the other hand, the disturbance response is written as follows

$$
\frac{Y(s)}{D(s)}=\frac{G_{p}(s)\left\{1-C_{m}(s) M(s)\right\}}{1+G_{p}(s) K(s)+G_{p}(s)}
$$

To eliminate the influence of an input side disturbance, we solve the following equations.

$$
\begin{aligned}
& \lim _{s \rightarrow 0}\left\{1-C_{m}(s) M(s)\right\}=0 \\
& \lim _{s \rightarrow 0} \frac{d}{d s}\left\{1-C_{m}(s) M(s)\right\}=0
\end{aligned}
$$

where, $M(s)$ is given by the following equation.

$$
M(s)=\frac{\beta_{1} s+\beta_{0}}{(\lambda s+1)^{2}}
$$




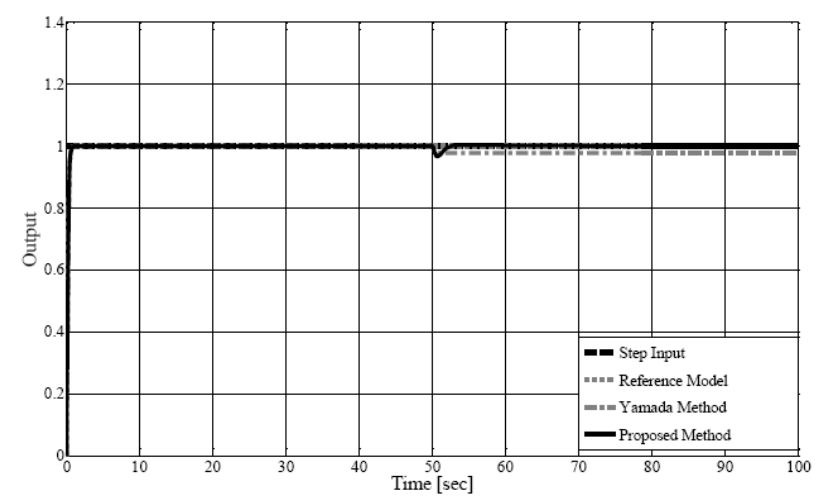

Fig. 3. Nominal plant

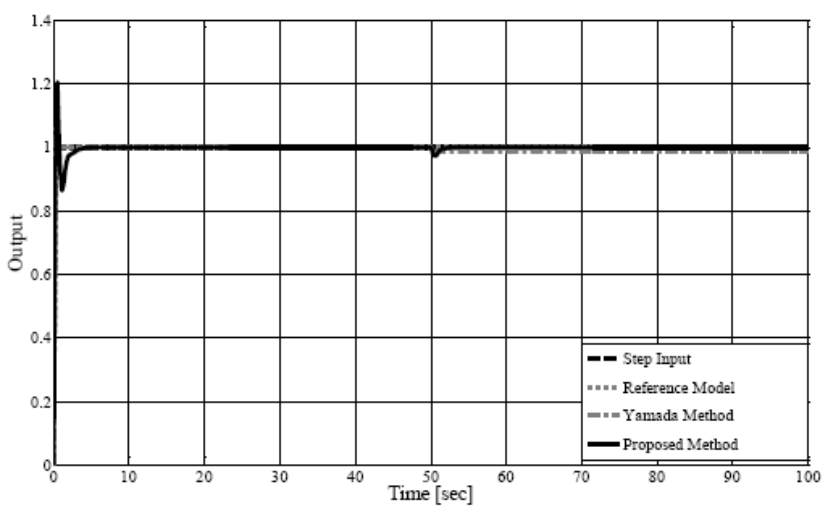

Fig. 4. Case 1

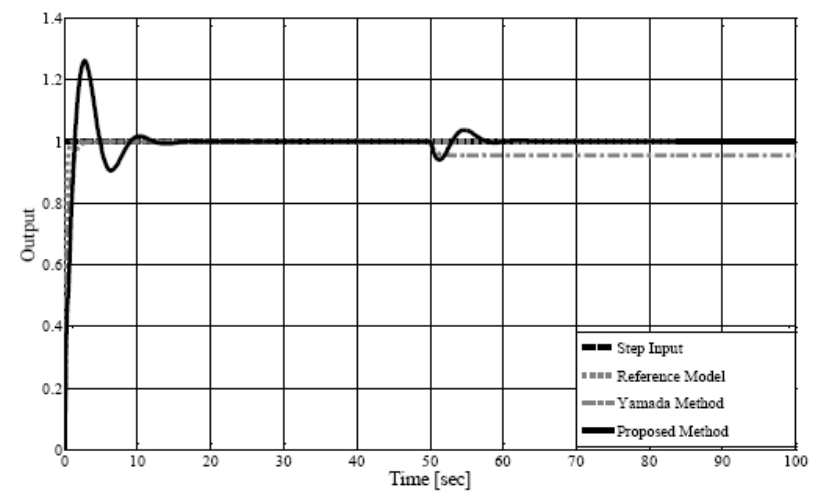

Fig. 5. Case 2

Parameters of the disturbance compensator in Eq.15 are given by Eq. 16 . Where $\lambda$ is a parameter to adjust influence by the disturbance and chosen from the following range; $0.5<\lambda<1.5$.

$$
\left\{\begin{array}{l}
\beta_{0}=1 \\
\beta_{1}=2 \lambda+\frac{2 \zeta}{\omega_{n}}
\end{array}\right.
$$

\section{Simulation Study}

We show simulation results of proposed method. Fig. 2 is a response of nominal plant and Fig. 3 is a response of the plant model with a modeling error as described later. An unit step is introduced at time $t=0$ [sec]. An input side disturbance $D(s)$ $=-1.0 / \mathrm{s}$ is introduced at time $t=50$ [sec]. An unstable plant with an integrator $G_{p}(s)$ and a stabilization controller $K(s)$ are written by the following equations. Therefore, $G(s)$ becomes a stable system.

$$
\begin{gathered}
G_{p}(s)=\frac{1}{s(s-1)} \\
K(s)=10 s \\
G(s)=\frac{1}{s(s+9)}
\end{gathered}
$$

\section{A. Nominal Plant}

The plant model has no modeling error.

$$
G_{m}(s)=\frac{1}{s(s-1)}
$$

A controller $C(s)$ and a disturbance compensator $M(s)$ are written as the following equations.

$$
\begin{aligned}
& C(s)=\frac{100 s^{2}}{s^{2}+20 s+100} \\
& M(s)=\frac{1.2 s+1}{(0.5 s+1)^{2}}
\end{aligned}
$$

\section{B. The plant model with a modeling error}

We describe the simulation results with two cases of modeling errors. The plant model $G_{m}(s)$ is given by Eq. 23 or Eq. 25 and a controller $C(s)$ is given by Eq. 24 or Eq. 26. In these cases, $M(s)$ does not depend on a plant model. Thus, $M(s)$ is the same equation: Eq. 22

Case 1:

The plant model has a modeling error: $+50 \%$

$$
\begin{aligned}
& G_{m}(s)=\frac{1.5}{s(s-1.5)} \\
& C(s)=\frac{150 s^{2}}{s^{2}+20 s+100}
\end{aligned}
$$

Case 2:

The plant model has a modeling error: $-50 \%$

$$
\begin{aligned}
& G_{m}(s)=\frac{0.5}{s(s-0.5)} \\
& C(s)=\frac{50 s^{2}}{s^{2}+20 s+100}
\end{aligned}
$$

\section{CONCLUSION}

In this paper, we have proposed a Modified Internal Model Control for an unstable plant with an integrator in continuous-time system. From simulation studies, it is shown that the proposed method has a superior performance. The nominal plant and the plant with a modeling error can be stabilized and the proposed method can reduce influence by an input side disturbance of an unstable plant.

\section{REFERENCES}

[1] M. Morari and E. Zafiriou, Robust Process Control, Prentice Hall, New Jersey, 1989.

[2] C. E. Garcia and M. Morari, "Internal Model Control-1. A unifying review and some new results," Ind. Eng. Chem. Process Des. \&Dev., vol. 21 , pp. 308-323, 1982. 
[3] C. E. Garcia and M. Morari, "Internal Model Control-2.Design Procedure for multivariable systems," Ind. Eng. Chem. Process Des. \&Dev., vol. 24, pp. 472-484, 1985.

[4] C. E. Garcia and M. Morari, "Internal Model Control-3.Multivariable Control low computation and tuning guidelines," Ind. Eng. Chem. Process Des. \&Dev., vol. 24, pp. 484-494, 1985.

[5] M. Oshima, Process Control System, Clona Publishing Co., Ltd, Tokyo, 2003.

[6] O. J. Smith, “A Controller to Overcome Dead Time," ISA Journal, vol. 6, pp. 28-33, 1959

[7] P. B. Despande and R. H. Ash, Computer process control, 2nd ISA Pub., 1988.

[8] K. J. Astrom, C. C. Hang, and B. C. Lim, "A Smith predictor for controlling a process with an integrator and long dead -time," IEEE Trans. On Automatic Control, vol. 39, pp. 343-345, 1994.

[9] I. Yoichi, M. Yuichi and I. Yoshihisa, "Discrete Modified Internal Model Control for plants with an integrator and time delay," IEICE Trans. On Fundamentals of Electronics, Communication and Computer Sciences, vol. J90-A, pp. 663-665, 2007.

[10] K. Yamada, "Modified Internal Model Control for unstable systems," in Proc. the 7th Mediterranean Conference on Control and Automation (MED99), pp. 293-302.

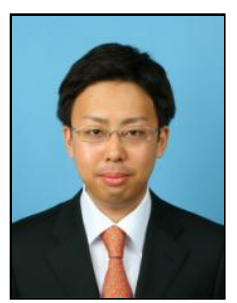

H. Shibasaki was born in Nov. 1988 in Tokyo, JAPAN. He received the B. E. degree in Electronics and Bioinformatics from Meiji University, Kawasaki, Japan, in 2011. He is currently studying toward the M. E. degree at Graduate School of Science and Technology, Meiji University. His research interests include continuous and digital control for a plant with integrator, and an unstable plant and its application to pneumatic equipment. He is a student member of the IEEE, and the IEEJ of Japan.

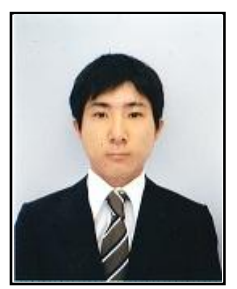

E. Junichi was born in Jul. 1988 in Tokyo, JAPAN $\mathrm{He}$ received the B. E. degree in Electronics and Bioinformatics from Meiji University, Kawasaki, Japan, in 2011. He is currently studying toward the M. E. degree at Graduate School of Science and Technology, Meiji University. His research interests include H-infinity control and its application to DC motor.

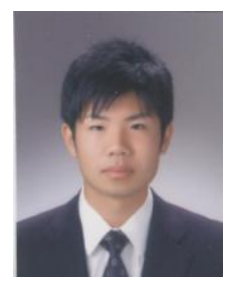

Y. Hikichi was born in Oct. 1988 in Kanagawa, JAPAN. He received the B. E. degree in Electronics and Bioinformatics from Meiji University, Kawasaki, Japan, in 2011. He is currently studying toward the M. E. degree at Graduate School of Science and Technology, Meiji University. His research interests include PID control and its application to linear motor.

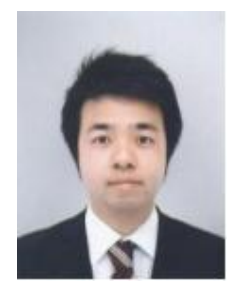

R. Tanaka was born in Oct. 1987 in Fukuoka, JAPAN. $\mathrm{He}$ received the B. E. degree in Electronics and Communications from Meiji University, Kawasaki, Japan, in 2010. He received the M. E. degree in Graduate School of Science and Technology, Meiji University, in 2012. He is currently studying toward the Dr. Eng. degree at Graduate School of Science and Technology, Meiji University. His research interests include Speech Signal Processing, Noise Reduction, and digital Control Systems.

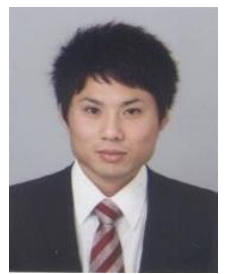

K. Kawaguchi was born in Feb. 1990 in Wakayama, JAPAN. He received the B. E. degree in Electronics and Bioinformatics from Meiji University, Kawasaki, Japan, in 2011 . He is currently studying toward the M. E. degree at Graduate School of Science and Technology, Meiji University. His research interests include Dahlin Algorithm for a plant with time delay and its application to pneumatic equipment.

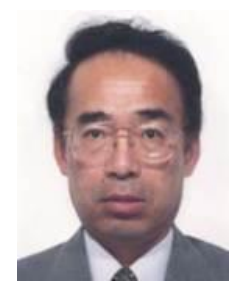

Y. Ishida was born in Feb. 1947 in Tokyo, JAPAN. He received the B. E., M. E., and Dr. Eng. Degrees in Electrical Engineering, Meiji University, Kawasaki, Japan, in 1970, 1972, and 1978, respectively. In 1975 he joined the Department of Electrical Engineering, Meiji University, as a research Assistant and became a Lecturer and an Associate Professor in 1978 and 1981, respectively. $\mathrm{He}$ is currently a Professor at the Department of Electronics and Bioinformatics, Meiji University. His current research interests include signal processing, speech analysis and recognition, and digital control. He is a member of the IEEE, and the IEICE of Japan. 